Chirurg 2009 $\cdot 80: 577-578$

DOI 10.1007/s00104-009-1755-3

Online publiziert: 28. Juni 2009

(c) Springer Medizin Verlag 2009

\author{
M.W. Büchler • M.N. Wente \\ Chirurgische Klinik, Universitätsklinikum Heidelberg
}

\section{Akute Appendizitis}

\section{Aktueller chirurgischer Stellenwert der Diagnostik und Standards der Therapie}

Pro Jahr werden in Deutschland ca. 130.00o Appendektomien durchgeführt. Damit gilt die Appendektomie als der häufigste chirurgische Notfalleingriff. Diese „kleine“ und oftmals von Chirurgen belächelte Operation hat ökonomisch und chirurgisch-fachlich im Sinne der Weiterbildung eine immense Bedeutung, insbesondere unter den Aspekten der Versorgung und der Versorgungsforschung. Es war den Herausgebern der Zeitschrift „Der Chirurg“ daher ein besonderes Anliegen, im vorliegenden Heft dieses Thema erneut aufzugreifen und nach den Kriterien der evidenzbasierten Medizin analytisch darzustellen.

Die Entfernung des entzündeten Wurmfortsatzes stellt in Deutschland einen unwidersprochenen Standard dar und dies auch aus medikolegaler Sicht. Diagnostisch gilt - so wie vor 100 Jahren - der klinische Symptomenkomplex aus Spontanschmerz im rechten Unterbauch und reproduzierbarem Druckschmerz in Verbindung mit Entzündungszeichen (Leukozytose, Fieber) als wegweisend [1] . Die Ultraschalluntersuchung hat sich in den vergangenen 20 Jahren mit einer Sensitivität und Spezifität von deutlich über $80 \%$ bei fehlender Strahlenbelastung als Standardbildgebung herauskristallisiert [2]. In unklaren Fällen kann die wesentlich aufwendigere Computertomographie zusätzliche Entscheidungshilfen geben [3].

Bei 10-15\% unserer Patienten werden nach wie vor "unschuldige“ Appendices entfernt [4]. Daher sind chirurgische Strategien zur Vermeidung negativer Appendektomien klinisch relevant und eine
Beschäftigung mit diesem für unsere Patienten wichtiger werdenden Thema ist angezeigt. Nach wie vor schlägt die zu strenge Indikationsstellung auf der ,anderen Seite" der häufigeren Perforationen zu Buche. Interessant ist in diesem Zusammenhang auch, dass die weitergehende histopathologische Untersuchung der Appendix unter Inanspruchnahme von immunhistochemischen Analyseverfahren bei einem Teil der in der primären HE-Untersuchung blanden Befunde noch das Ergebnis einer speziellen schmerzverursachenden chronischen Inflammation erbringt [5]. Im Einzelfall ist daher vom Pathologen die weitergehende Untersuchung der „blanden“ Appendix zu fordern.

\section{$\checkmark$ Die laparoskopische Appendektomie ist auf dem Vormarsch}

Die beste Strategie zur Vermeidung einer negativen Appendektomie ist der klinisch erfahrene Chirurg, der unter Zuhilfenahme von Standardlaboruntersuchungen (Leukozyten, CRP) und einem bildgebenden Verfahren (Ultraschall) den Patienten hospitalisiert und ihn bei vorhandenen Kriterien zeitnah operiert bzw. im Verlauf von $24 \mathrm{~h}$ die Indikation stellt oder verwirft. Die Entfernung einer makroskopisch unauffälligen Appendix ist in jedem Falle unproblematisch, wenn das im präoperativen Aufklärungsgespräch mit dem Patienten vereinbart wurde.

Die laparoskopische Chirurgie zur Therapie der akuten Appendizitis ist in Deutschland ohne Zweifel auf dem Vormarsch, obwohl die aktuellen landesweit registrierten Zahlen eine 50:50 Verteilung offene Appendektomie vs. laparoskopische Appendektomie zeigen. Im eigenen Heidelberger Krankengut ist die Rate der laparoskopischen Wurmfortsatzentfernung bei $96 \%$ angekommen. In randomisierten Studien und Metaanalysen ist der Vorteil der laparoskopischen Appendektomie hinsichtlich Schmerzverminderung, geringerer Wundinfektionsrate, besserer Kosmetik, schnellerer Krankenhauserholung und Wiedereintritt in das Berufsleben belegt; die Nachteile liegen in einer Erhöhung der Rate intraabdomineller Abszesse und auf der Seite der höheren Krankenhauskosten (ca. 20\% durch höhere Materialkosten) [6, 7].

Die laparoskopische Revolution hat sich bei der Appendektomie langsamer vollzogen als bei der Cholezystektomie. Es ist jedoch davon auszugehen, dass das Pendel in den nächsten 10 Jahren eindeutig zugunsten der laparoskopischen Therapie ausschlagen wird, zumindest wird sie immer häufiger eingesetzt, allerdings mit leicht höheren Komplikationsraten, wenn man auf aktuelle Ergebnisse der Versorgungsforschung zurückgreift [8]. Die offene Appendektomie ist ohne Zweifel und vor allem auch aus medikolegaler und ökonomischer Sicht weiterhin gleichberechtigt einsetzbar, insbesondere wenn kein laparoskopisch erfahrener Chirurg im jeweiligen Nachtdienst anwesend ist.

Die vorliegenden vier Übersichtsartikel aus Aachen, Berlin und Heidelberg reflektieren eindrucksvoll die aktuellsten wissenschaftlichen und klinischen Daten zur Diagnostik und Therapie dieser häufigsten Notfalloperation. Ein neuer Kapi- 
tän (Laparoskopie) ist an Bord, ob er den Tanker (130.00o Operationen) mit ruhiger Hand in die richtige Richtung lenkt, wird die Zukunft zeigen. Man traut es ihm $\mathrm{zu}$.

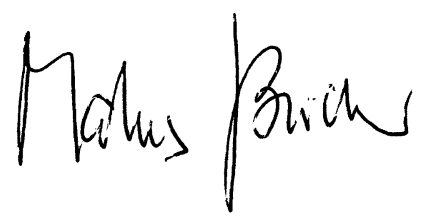

M. W. Büchler

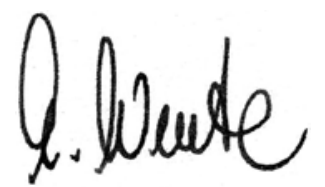

M. N. Wente

\section{Korrespondenzadresse \\ Prof. Dr. M.W. Büchler \\ Chirurgische Klinik, \\ Universitätsklinikum Heidelberg \\ Im Neuenheimer Feld 110, 69120 Heidelberg \\ Markus.Buechler@med.uni-heidelberg.de}

\section{Literatur}

1. Andersson RE (2004) Meta-analysis of the clinical and laboratory diagnosis of appendicitis. Br J Surg 91:28-37

2. Doria AS, Moineddin R, Kellenberger CJ et al (2006) US or CT for diagnosis of appendicitis in children and adults? A meta-analysis. Radiology 241:83-94

3. Van Breda Vriesman V, Kole BJ, Puylaert JB (2003)

Effect of ultrasonography and optional computed tomography on the outcome of appendectomy. Eur Radiol 13:2278-2282

4. Flum DR, Morris A, Koepsell T, Dellinger EP (2001) Has misdiagnosis of appendicitis decreased over time? A population-based analysis. JAMA 286:1748-1753

5. Di Sebastiano P, Fink T, di Mola FF et al (1999) Neuroimmune appendicitis. Lancet 354:461-466

6. Chung RS, Rowland DY, Li P, Diaz J (1999) A metaanalysis of randomized controlled trials of laparoscopic versus conventional appendectomy. Am J Surg 177:250-256

7. Sauerland S, Lefering R, Neugebauer EA (2004) Laparoscopic versus open surgery for suspected appendicitis. Cochrane Database Syst Rev CD001546

8. Sporn E, Petroski GF, Mancini GJ et al (2009) Laparoscopic appendectomy-is it worth the cost? Trend analysis in the US from 2000 to 2005. J Am Coll Surg 208:179-185

\section{Themenübersicht}

"Der Chirurg“ bietet Ihnen jeden Monat umfassende und aktuelle Beiträge zu interessanten Themenschwerpunkten aus allen Gebieten der operativen Medizin.

Möchten Sie ein bereits erschienenes Heft nachbestellen? Die folgenden Ausgaben können Sie direkt bei unserem Kundenservice zum Preis von je EUR 32,- beziehen:

Wir haben die Jahrgänge 2008/2009 im Überblick für Sie zusammengestellt:

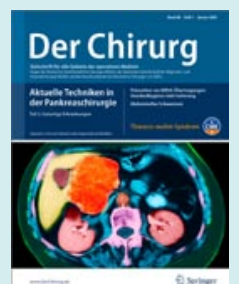

2008

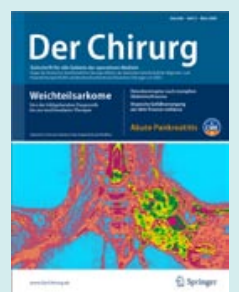

- $1 / 2008$ Chirurgie des Mediastinums

- 2/2008 Lebertransplantation

- 3/2008 Allgemeinchirurgie in der Diskussion

- 4/2008 Peritonitis

- 5/2008 Aktuelle Proktologie

- 6/2008 Die chronische Wunde

- 7/2008 Gastrointestinale Stromatumoren

- 8/2008 Benigne Lebertumoren inkl. Echinococcus

- 9/2008 Morbide Adipositas

- 10/2008 Wirbelsäulenchirurgie

- 11/2008 Hereditäre Karzinome

- 12/2008 Aktuelle Techniken in der

Pankreaschirurgie - Teil 1

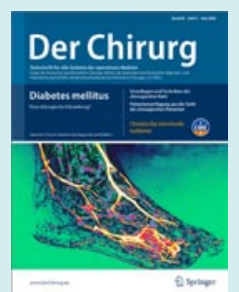

2009

- 1/2009 Aktuelle Techniken in der Pankreaschirurgie - Teil 2

- 2/2009 Neue Konzepte in der Endokrinen Viszeralchirurgie

- 3/2009 Weichteilsarkome

- 4/2009 Rektumkarzinom

- 5/2009 Diabetes mellitus

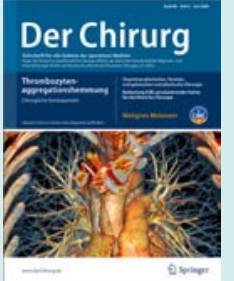

- 6/2009 Thrombozytenaggregationshemmung

- 7/2009 Akute Appendizitis

- 8/2009 Fast Track in der Chirurgie

- 9/2009 Komplikationsmanagement in der Chirurgie

- 10/2009 Lebertrauma

- 11/2009 Oberer Gastrointestinaltrakt

- 12/2009 Jubiläumsheft - 80 Jahre Der Chirurg

\section{So erreichen Sie unseren Kundenservice:}

Springer Customer Service Center GmbH

Haberstr. 7

69126 Heidelberg

Tel.: +49 $6221345-4303$

Fax: +496221 345-4229

E-Mail: Leserservice@springer.com

\section{www.DerChirurg.de}

